\title{
Investigation médicale et juridique de cas de responsabilité civile du médecin
}

\author{
$3^{\text {e }}$ partie: exemples tirés de la pratique - règlement des cas de dommage sans expertise
}

W. E. Ott

Deutsch erschienen

in Nr. 13/2004

1 Le lecteur trouvera des exemples de réalisation d'un risque général, ainsi que de plus amples explications, dans l'ouvrage de casuistique de Ott WE. Fehlerhafte Unfallbehandlung, p. 458 s.
Dans cette catégorie, nous trouvons tout d'abord les cas médicaux dont l'élucidation, effectuée par les deux parties, débouche sur un résultat favorable au patient, tant par rapport à la présence d'une violation du devoir de diligence ou d'information (voire les deux), que par rapport à la causalité. Une expertise indépendante n'a alors plus de raison d'être.

Nombre de cas d'atteintes à la santé ne sauraient néanmoins déboucher sur une appréciation aussi claire. Si des motifs sérieux indiquent tout de même que l'assistance médicale n'a pas été entièrement apportée selon les règles de l'art, l'assurance responsabilité civile a la possibilité, nous l'avons évoqué, de procéder à un «achat du risque»: sans créer de précédent, donc sans se soumettre à un devoir juridique, elle met à disposition une somme d'indemnisation unique et forfaitaire tenant compte des risques bilatéraux en cas d'extension de l'affaire et permettant de parvenir le plus vite possible à un règlement à l'amiable. Une telle solution ne permet pas seulement d'éviter des dépenses et un investissement de temps supplémentaires: le patient peut lui aussi y trouver un avantage puisqu'il doit malgré tout s'attendre, en cas d'expertise défavorable pour lui, à devoir s'en aller les mains vides.

Enfin, lorsque l'enquête médicale prouve clairement que ses critiques ne tiennent pas face à un examen objectif et rendent inutiles des démarches ultérieures, le patient doit subir le dommage sans indemnisation aucune. Il va sans dire qu'il s'agit là de circonstances difficiles à vivre pour lui [1].

L'auteur présente les exemples qui suivent sans commentaires. Des réflexions supplémentaires dépasseraient en effet le cadre du présent article, ce qui ne veut pas dire que certains des cas cités ne soient pas dignes de débat.

\section{Accord à l'amiable (pas de précédent pour les deux parties, question de la responsabilité laissée ouverte)}

Cas $\mathrm{n}^{\circ} 1$

Me Werner E. Ott, Dr en droit Badenerstrasse 21

CH-8026 Zurich
Faute de diagnostic - carcinome bronchique visible au lobe pulmonaire supérieur droit, non décelé lors d'un contrôle radiologique routinier du thorax. Résection du lobe, chimiothérapie, métastases aux ganglions lymphatiques. Situation typique de «perte d'une chance». Versement d'une indemnité.

\section{Cas $\mathbf{n}^{\circ} 2$}

Faute de diagnostic - récidive non diagnostiquée - carcinome après amputation du sein; opération de révision et traitement ultérieur. L'apport de preuves est rendu impossible par une perte inexpliquée des radiographies. Accord à l'amiable.

\section{Cas $\mathbf{n}^{\circ} 3$}

Faute de diagnostic - grossesse extra-utérine non décelée à temps. Problèmes de communication entre médecin et patiente, hospitalisation en urgence, déroulement avec complications sans suites permanentes. Accord à l'amiable.

\section{Cas $n^{\circ} 4$}

Faute de diagnostic - diagnostic tardif d'une torsion testiculaire chez un enfant de 15 ans, nécrose testiculaire, orchidectomie gauche. Cas insuffisamment éclairci (examen insuffisant et/ou problème de communication): accord à l'amiable.

\section{Cas $\mathrm{n}^{\circ} 5$}

Faute de diagnostic - rupture ignorée du tendon d'Achille après un accident de sport. Mise au repos pendant des semaines avec attelle et antalgiques. Un simple test métatarso-phalangien ou une échographie auraient été de bon conseil. Opération tardive, guérison tardive, entière couverture des frais.

\section{Cas $\mathrm{n}^{\circ} 6$}

Faute de traitement - appendicectomie retardée. A l'hospitalisation, paramètres de l'inflammation nettement augmentés. Diagnostic CT: appendicite avec inflammation des tissus graisseux environnants. Opération 16 heures plus tard seulement. Appendicite aiguë gangréneuse perforée. Péritonite, déroulement non sans complications. Accord à l'amiable. 


\section{Cas $\mathrm{n}^{\circ} 7$}

Faute de traitement - vasectomie avec spermiogramme positif. En dépit de trois spermiogrammes postopératoires positifs, l'opérateur permet les relations sexuelles sans produit anticonceptionnel. Faute avérée. L'épouse est à nouveau enceinte. Naissance d'un enfant sain. Pas de «dommages» au sens de la doctrine traditionnelle, mais octroi tout de même d'une somme forfaitaire.

\section{Cas $\mathrm{n}^{\circ} 8$}

Faute de traitement - examen tomographique: perte de l'audition et tinnitus provoqués par des écouteurs mal placés. Instruction et surveillance insuffisantes de la part d'une assistante. Accord à l'amiable.

\section{Cas $\mathrm{n}^{\circ} \mathbf{9}$}

Assistance en urgence insuffisante - écrasement des phalanges terminales de deux doigts de la main droite. Traitement ambulatoire, pas d'hospitalisation pour surveiller l'évolution ni de contrôle final, nécrose étendue des phalanges, amputation. Accord à l'amiable.

\section{Cas $\mathrm{n}^{\circ} 10$}

Complication anesthésique - arthroscopie du coude gauche en anesthésie du plexus. L'anesthésique a pénétré partiellement dans les voies sanguines, ce qui a conduit à un trouble fonctionnel invalidant du mésencéphale. Indemnisation sous forme de couverture des frais et réparation pour tort moral subi par le conjoint.

\section{Cas $\mathrm{n}^{\circ} \mathbf{1 1}$}

Complication anesthésique - intervention gynécologique. Le mode anesthésique, inapproprié, a provoqué un œdème des cordes vocales avec enrouement permanent. Narcose par intubation en dépit d'une situation anatomique étroite. Antibiothérapie erronée. Dommage permanent. Accord à l'amiable.

\section{Cas $\mathrm{n}^{\circ} 12$}

Complication opératoire et anesthésique - chute dans l'escalier avec rupture tendineuse de l'annulaire. Probablement analgésie insuffisante avec nécessité de réinjecter, évent. dommage du nerf ulnaire distal du bras par blocage sanguin. Situation peu claire et documentation lacunaire. Accord à l'amiable.

\section{Cas $\mathrm{n}^{\circ} 13$}

Incident lors de l'injection d'un produit de contraste. Paravasat, injection paraveineuse non remarquée d'une partie du produit de contraste, intervention d'urgence nécessaire. Fonction limitée de l'articulation de la main. Prise en charge du dommage direct non couvert et octroi d'une indemnité (la reconnaissance, médicale et juridique, des complications du Paravasat est régulièrement contestée).

\section{Cas $n^{\circ} 14$}

Incident d'injection - épicondylite, infiltration locale i.m. (1 ampoule de Diprophos ${ }^{\odot}$, combinée avec du Carbostetin ${ }^{\odot}[0,5]$ et une ampoule de Voltaren $^{\odot}$ ). Syndrome du supinateur, suite à une lésion du nerf interosseux postérieur par une injection probablement effectuée de manière inappropriée. Accord à l'amiable.

\section{Cas $\mathrm{n}^{\circ} 15$}

Erreur de groupe sanguin - réthoracotomie au cours de laquelle le sang d'un mauvais groupe a été transfusé. Grave réaction transfusionnelle hémolytique. Carte de groupe sanguin mal remplie au laboratoire. Prise en charge totale du dommage (passager).

\section{Cas $\mathrm{n}^{\circ} 16$}

Faute de traitement - inflammation et nécrose dans la région du dos du pied par perfusion paraveineuse. Prise en charge totale du dommage et versement d'une indemnité.

\section{Cas $\mathrm{n}^{\circ} 17$}

Faute opératoire - césarienne au cours de laquelle une suture, par inadvertance, a été pratiquée entre les lèvres antérieure et postérieure de l'orifice externe de l'utérus au lieu d'entre la paroi vaginale abaissée et la paroi utérine antérieure. Sévère septicémie, risque mortel, longue convalescence. Prise en charge totale du dommage.

\section{Cas $\mathrm{n}^{\circ} 18$}

Faute opératoire - refracture d'une fracture fémorale insuffisamment refermée après élimination de l'implant et enclouage gamma de la hanche gauche. Suite à l'hypothèse qu'un clou d'ostéosynthèse intramédullaire probablement trop long irrite la région opérée, élimination trop précoce du matériel de l'implant et oubli de consolider la fracture - considérée à tort comme stable à l'examen radiologique - au moyen d'un clou moins long. Octroi d'une somme à titre de frais et indemnité.

\section{Cas $\mathrm{n}^{\circ} 19$}

Faute opératoire - ostéosynthèse d'une fracture par torsion du métacarpe IV droite; au cours de l'opération, section non remarquée du tendon extenseur du quatrième os du métacarpe. 
Déficit fonctionnel en dépit de la reconstruction. Prise en charge totale du dommage.

\section{Cas $\mathrm{n}^{\circ} \mathbf{2 0}$}

Faute opératoire - suite à une identification insuffisante et à une confusion, ablation d'une exostose au mauvais endroit, défaut osseux restant à l'endroit opéré de la partie dorsale du condyle fémoral médian. Deuxième opération nécessaire pour résection de l'exostose. Prise en charge totale du dommage.

\section{Cas $\mathrm{n}^{\circ} 21$}

Faute opératoire et mauvaise assistance postopératoire. Mauvaise suture, péritonite après résection laparoscopique du sigmoïde. Un dysfonctionnement du drainage a provoqué une fuite décelée trop tard et lourde de conséquences. Assistance hospitalière limitée en raison des jours de fête. Faute d'organisation (?). Accord à l'amiable.

\section{Cas $\mathrm{n}^{\circ} \mathbf{2 2}$}

Faute opératoire et faute de suivi - plastie mammaire de réduction avec caractère de maladie, déhiscence de la suture, infection postopératoire, traitement insuffisant. Plusieurs opérations ont dû suivre avec pour conséquence une reconstruction mammaire au silicone en raison du manque total de tissu mammaire. Indemnité et couverture des frais.

\section{Cas $\mathrm{n}^{\circ} 23$}

Faute de suivi - infection après arthroscopie du ménisque; intervention ambulatoire dans un cabinet privé, grave infection de source indéterminée. Complications, risques mortels, 10 jours en unité de soins intensifs. Difficile investigation rétrospective pour déterminer si les prescriptions sévères en matière de stérilité ont été respectées. Les preuves «prima facie» semblent montrer que ce n'est pas le cas. Accord à l'amiable.

\section{Cas $\mathrm{n}^{\circ} 24$}

Faute de traitement - intervention dermatologique, élimination d'une cicatrice de varicelle dans le décolleté, chez une patiente encline à former des chéloïdes. Résultat très insatisfaisant, aggravé par d'autres révisions cicatricielles. Une intervention à cet endroit ne serait indiquée qu'en cas de modification tissulaire cancéreuse. Accord à l'amiable.

\section{Cas $n^{\circ} 25$}

Surveillance postopératoire insuffisante - plastie laparoscopique d'une hernie inguinale des deux côtés, mauvais déroulement postopératoire avec fortes douleurs abdominales et signes de choc insuffisamment observés, abdomen septique, iléus d'adhésion, aggravation sous-estimée. Intervention retardée dans la cavité abdominale, élimination d'une partie d'intestin nécrotique de 1,5 $\mathrm{m}$ de long. Troubles multiorganiques, risques mortels, plusieurs opérations en urgence nécessaires. Longue convalescence. Invalidité permanente. Prise en charge totale du dommage.

\section{Cas $n^{\circ} 26$}

Prothèse de substitution trop tardive - implantation d'une prothèse de la hanche; malgré un avertissement ultérieur du fabricant sur des problèmes de matériel, on s'est contenté d'effectuer des contrôles annuels et de faire de la physiothérapie. Pas de descellement de la prothèse, mais dommage osseux considérable. Remplacement objectivement trop tardif d'une prothèse sans doute défectueuse. Longue période de douleurs. Accord à l'amiable.

\section{Cas $\mathrm{n}^{\circ} \mathbf{2 7}$}

Faute de soins - déchargement d'une patiente avec fracture du bras hors de l'ambulance, transfert sur une civière. La civière se renverse. La patiente tombe sur le sol en béton. Aggravation supposée de la fracture (fracture multiple). Traitement plus difficile. Convalescence plus longue. Accord à l'amiable.

\section{Cas $\mathrm{n}^{\circ} \mathbf{2 8}$}

Faute de soins - opération en urgence d'une rupture du talon d'Achille. Pose d'une attelle Fractomed. Brûlures iatrogènes au $2^{\mathrm{e}}$ degré de la jambe droite. Dommage esthétique. Indemnité et remboursement des frais.

\section{Affaires sans suite (pas de précédent, question de la responsabilité laissée ouverte)}

\section{Cas $\mathrm{n}^{\circ} 29$}

Pas de faute de diagnostic - diagnostic tardif d'un lymphome non hodgkinien, très malin, à grandes cellules B. Les investigations ont révélé qu'au moment de l'hospitalisation d'urgence et du diagnostic, le stade de la maladie était à ce point avancé que seul un traitement palliatif était possible. Le médecin généraliste n'avait pas la possibilité de réagir plus tôt. En effet, en raison des symptômes apparus trois semaines après le début du traitement, le médecin a annoncé le patient pour une gastroscopie qui n'a plus pu avoir lieu. Décès rapide. Dossier classé sans suite. 


\section{Cas $\mathrm{n}^{\circ} \mathbf{3 0}$}

Pas de faute de diagnostic et/ou de traitement crise de migraine supposée avec maux de tête violents, résistant au traitement. Refus d'hospitalisation de la part du patient, ainsi que des investigations recommandées pour un diagnostic différencié. Décès du patient d'une défaillance de la régulation centrale en raison d'une hémorragie due à une rupture d'anévrisme de l'artère basique moyenne du cerveau droit. Dossier classé sans suite.

\section{Cas $\mathrm{n}^{\circ} 31$}

Pas de faute opératoire - reposition ouverte et ostéosynthèse d'une fracture distale du radius en plusieurs fragments, plastie des tissus spongieux. Malposition s'en suivant et apparition d'une maladie de Sudeck, tableau clinique multifactoriel, intervention de révision non indiquée tant que ce problème subsiste. Dossier classé sans suite.

\section{Cas $\mathrm{n}^{\circ} 32$}

Pas de faute opératoire - perforation de l'intestin après cholécystectomie laparoscopique. Même à l'examen microscopique, la cause de la lésion de la paroi intestinale ne peut être imputée avec haute probabilité à un dommage intraopératoire. Dossier classé sans suite.

\section{Cas $\mathrm{n}^{\circ} 33$}

Pas de diagnostic de grossesse manqué - consultation en raison de troubles de l'hypogastre. Infection, rendez-vous pour un contrôle, problèmes de communication. Selon le dossier de la patiente, le médecin communique le diagnostic de grossesse, que la patiente n'a probablement pas compris. Documentation médicale sans lacunes, y compris en ce qui concerne les résultats de laboratoire et d'ultrasonographie. Dossier classé sans suite. 